\author{
MARIAN SZCZODROWSKI \\ Ateneum Szkoła Wyższa w Gdańsku
}

\title{
Zur Entwicklung der kodematischen Theorie des Fremdsprachenunterrichts
}

\section{On the development of the codematic theory of foreign-language learning}

\begin{abstract}
The discussion addresses the linguistic code and its contribution to the development of the codematic theory of foreign-language teaching. This theory deals with the essence and properties of the course of processes of coding and decoding foreign-language structures. Teachers and learners participate in these processes as primary and secondary senders and receivers of transmitted lexical-grammatical structures with the aim of learners' mastering them. The process of learning and mastering foreign-language structures takes place in two stages, that is, during their decoding and construction in the form of structural matrices in the receiver's appropriate memory. In this way, in the receiver (learner) there forms a warehousing mechanism with regard to the foreign language. This mechanism is equipped with a receptive and (re)productive functioning force, that is a force that activates the structural matrices. Structural matrices in long-term memory attain the appropriate force of activation, and therefore demand constantly being brought up to date in various syntagmatic-syntactic constructions, that is in the process of their encoding.

KEYWORDS: linguistic code, foreign-language code processes, primary and secondary sender, primary and secondary receiver, merging structural matrices, spoken and written linguistic structural matrices and their processes of encoding.

SCHLÜSSELWÖRTER: Sprachkode, fremdsprachliche Kode-Prozesse, Primärsender und Sekundärsender, Primärempfänger und Sekundärempfänger, Verkoppelung der Strukturmatrizen, Sprech-SchreibSprach-Strukturmatrizen und ihre Einkodierungsprozesse.
\end{abstract}

\section{EINLEITUNG}

Für den Fremdsprachenunterricht sind verschiedene wissenschaftliche Theorien ausgearbeitet worden, die als begründete Darstellungen erklären und vorschlagen, wie sie didaktisch-methodisch in der Lehr-Lern-Praxis umgesetzt werden können. Zwischen den linguistischen Theorien, welche die 
Sprachsysteme hinsichtlich deren Funktion in den Kommunikationsvorgängen untersuchen und beschreiben, und den fremdsprachenunterrichtlichen Theorien, welche die Erlernungs- und Aneignungsprozesse der Fremdsprache(n) erörtern, didaktisch-methodische Konzeptionen ausarbeiten und vorlegen, bestehen enge Relationen.

In diesem Beitrag versuchen wir, auf das Wesen des Sprachkodes und seine Relevanz auf die Entwicklung der kodematischen Theorie des Fremdsprachenunterrichts hinzuweisen und zu schildern, welche Subprozesse den fremdsprachlichen Kodierungs- bzw. Dekodierungsprozess konstituieren und wie diese ablaufen. Darüber hinaus werden noch den neuen dekodierten fremdsprachlichen Strukturen und deren im Gedächtnis aufbewahrten Sprachmatrizen besondere Aufmerksamkeit gelenkt, dank denen sie länger im Speicher-Mechanismus behalten werden können. Diese Ziele erreicht man auf Grund der Einprägung dieser Sprach-Strukturmatrizen in die tieferen Schichten des Langzeit-Gedächtnisses und solche Prozesse bezeichnen wir - der kodematischen Theorie gemäß - als Einkodierungsprozesse.

\section{SPRACHKODE UND SEINE SPEZIFIK}

Wird die Begrifflichkeit des Kommunikationskodes eingehender ergründet, so zeigt sich, dass in seinem Bereich mehrere Sonderkodes funktionieren, die in zwei Grundsysteme, und zwar in die sprachlichen und die nichtsprachlichen Kodes eingeteilt werden können (Abb. 1).

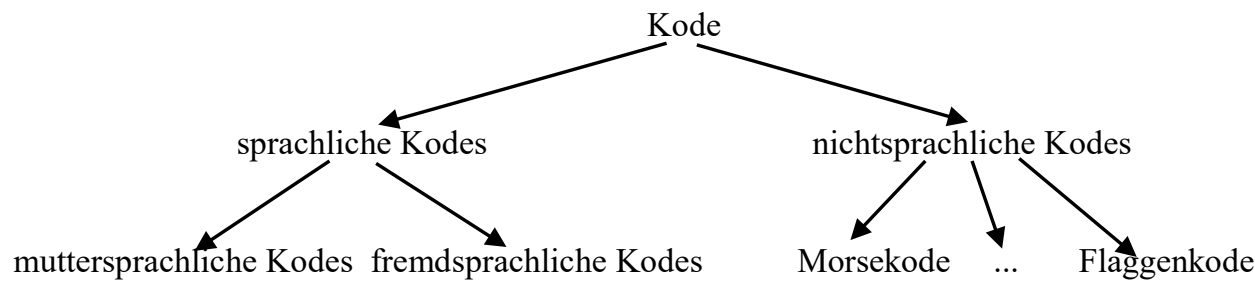

Abbildung 1. Kodes und ihre Arten

Wie aus dem obigen Schema ersichtlich sein dürfte, gilt der Kode als Sammelbegriff für verschiedene Arten menschlicher Kommunikation. Sein Wesen schließt das in der Kommunikationsgemeinschaft vereinbarte und regelhafte Inventar von Zeichen oder Signalen ein, mittels deren Informationen zwischen den Kommunikationspartnern, die den gleichen Kode verwenden, übertragen werden (vgl. Baacke 1973: 95 u. 88; Gipper 1978: 173). Betrachtet man den 
Kodebegriff im Lichte der Informations- und Kommunikationstheorie, wo die Gesetzmäßigkeiten der Übertragung von Informationen mit mathematischen Methoden untersucht werden, so lässt sich das dort verwendete Sender-Empfänger-Modell für die Sprachkommunikation adaptieren und die durch den Sender (Sprecher/Schreiber) und den Empfänger (Hörer/Leser) benutzte Sprache als Kode bezeichnen. Dementsprechend wird der im Sender generierende Vorgang von Informationen als Kodierung und der sich im Empfänger vollziehende Aufnahmevorgang der Informationsstrukturen als Dekodierung definiert. Die Kommunikationspartner sind über den Kanal verbunden, durch den die vom Sender kodierten Informationen an den Empfänger vermittelt werden. Dieses Faktum zeugt deutlich davon, dass die Kommunikation unbedingt der Information(en) bedarf, d. h. die Kommunikation bedingt die Information oder noch anders ausgedrückt: sie stehen in gegenseitiger Abhängigkeit (vgl. Bünting \& Kochan 1973: 93ff.).

Jeder einzelne Sprachkode hat sich in der bestimmten Sprachgemeinschaft so herausgebildet, dass seine phonetisch-phonologischen und graphisch-graphematischen Strukturen (falls der Kode über sie verfügt) sowie seine lexikalischen und grammatischen Strukturen ein geordnetes Gefüge darstellen. Diese Strukturen wiederholen sich - entsprechend den in der Sprache festgelegten Gesetzmäßigkeiten - in Verbindungen verschiedener Art und bilden das sprachliche System als Grundlage für die zwischenmenschliche Verständigung. Im Bereich des sprachlichen (allgemeinen) Systems werden weiterhin dessen Subsysteme und auch Subsubsysteme unterschieden.

Eine wissenschaftlich gründliche Analyse des Sprachkodes hat Ludwik Zabrocki (1961: 65ff.) durchgeführt. Ausgehend vom allgemeinen Kodebegriff, der als Gesetz der Transponierbarkeit von irgendwelchen Zeichen in andere Zeichen definiert wird, deutet Zabrocki auf die Gesetze der Transponierbarkeit der dem Begriff des Sprachkodes zugrunde liegenden sprachlichen Zeichen hin. Bezugnehmend auf die Konzeption des bilateralen Charakters des sprachlichen Zeichens wird die Funktion der Transponierbarkeit des Kodes im Bereich der bezeichnenden Substanz hervorgehoben, wo sie durch eine andere bezeichnende Substanz des Zeichens repräsentiert werden kann. Der Transponierungskode bezieht sich nur auf die bezeichnende Substanz, die bezeichnete Substanz des Inhaltsplans unterliegt jedoch nicht dem Transponierungsprozess, wodurch sie ihre inhaltliche Substanz ohne jede Veränderung beibehält. In weiteren Erörterungen unterscheidet Zabrocki den Lauttransponierungs-Kode und den Morphemtransponierungs-Kode. Er erläutert das Wesen und die Funktion des synthetischen Transformations-Kodes, auf Grund dessen die Worteinheiten im Satz angeordnet werden, und des analytischen Transformations-Kodes, auf Grund dessen die syntaktische Struktur in Syntagmen und Worteinheiten zergliedert wird. 


\section{FREMDSPRACHLICHE KODE-PROZESSE}

In den Arbeiten, die die fremdsprachlich-kodematische Problematik aufgreifen, werden zwei Kode-Grundprozesse dargestellt und erläutert, und zwar des Kodierungs- und des Dekodierungsprozesses. Da der Sprachkode für die zwischenmenschliche Kommunikation relevant ist, wird dessen Funktion eo ipso in den Lehr-Lern-Prozessen berücksichtigt. Gegenstand unserer Erörterungen sind Kode-Prozesse, die in den institutionalisierten (hier schulischen) Formen des Fremdsprachenunterrichts stattfinden. In diesem Falle handelt es sich um Deutsch als Fremdsprache. Die Sprache wird hier unter anderen Bedingungen gelernt und erworben als im sprachlichen Unterricht von Deutsch als Muttersprache. Die Lernenden, die sich unter schulischen Bedingungen die Fremdsprache aneignen, beherrschen bereits ihre Muttersprache, die als Ausgangssprache betrachtet wird, und die den fremdsprachlichen Lernprozess einerseits zwar erleichtern, andererseits aber auch erschweren kann.

Ludwik Zabrocki (1966: 3-42) hat die kodematischen Grundlagen für die Theorie des Fremdsprachenunterrichts gelegt und gilt auch als Begründer des kybernetischen Strukturalismus, wo die Sprache als Tätigkeit, Wirkung (Energeia) in das kybernetische Gefüge der Kommunikationsprozesse eingebettet wird (vgl. Helbig 1970: 344f.). In den theoretischen Erörterungen hebt Zabrocki (1966: 9) ausdrücklich hervor, dass man den Sprachkode im Sprechprozess als entsprechende Transformationsregeln bezeichnen kann, die der Kodematik zugrunde liegen. Für die kodematische Theorie des Fremdsprachenunterrichts ist von besonderer Wichtigkeit, dass sich der Lehrende des für die zu lernende Fremdsprache bestimmten Transformationskodes bedient. Dementsprechend taucht das didaktisch-methodische Problem auf, wie die Transformationsregeln den Lernenden erklärt und vermittelt werden, damit diese sie erfolgreich begreifen und sich aneignen können. Mit diesem Problem ist noch ein anderes verbunden, nämlich die außersprachliche Wirklichkeit, deren Bestandteile (Gegenstände, Erscheinungen und ihre Eigenschaften) in jeder Sprache auf eine spezifische Art und Weise abgebildet, segmentiert und dabei unterschiedlich kodiert werden, was auch den Fremdsprachenlernern Schwierigkeiten bereiten kann. Die fremdsprachenunterrichtliche Kommunikation wird von Zabrocki (1966: 15 u., 1975: 26) in das allgemeine Sprachkommunikationsgefüge eingebaut, das des Weiteren in das kybernetische Gefüge eingeordnet wird, wo man es mit dem Sender und dem Empfänger, der/den Information(en) und den Übertragungskanälen zu tun hat. Unter den Kanälen sind zu unterscheiden: 1. der nukleare Kanal, durch den die vom Sender kodierten Informationen an den Empfänger vermittelt werden, 2. der Steuerungskanal, der für die optimale und erfolgreiche Übertragung der Informationen verantwortlich ist, und 3. der 
Kontrollkanal, durch den die Erfolge des sich im Primärempfänger vollzogenen Dekodierungsprozesses der Informationsstrukturen nach entsprechenden Transformationen vom Sekundärsender kodiert und dem Primärsender mitgeteilt werden. Daraus ergibt sich, dass die fremdsprachlichen Kommunikationspartner sowohl einseitig als auch im Rückkoppelungsgefüge miteinander verbunden sind. Den materiellen Kommunikationsvermittler bilden die lautliche Substanz (die phonetisch-phonologischen Strukturen) oder die schriftliche Substanz (die graphisch-graphematischen Strukturen).

Den Fremdsprachenunterricht beginnt selbstverständlich der Lehrende/Sender, der die bestimmten Informationsstrukturen den Lernenden/Empfängern vermittelt, die sie dekodieren, kennenlernen, verstehen und behalten. Dementsprechend ist unsere Aufmerksamkeit insbesondere auf die Lernenden gelenkt. Es ist wichtig zu wissen, wie sie diese fremdsprachliche Substanz aufnehmen, dekodieren und speichern. Die Handlungen verlangen genauere Erörterungen, die von Zabrocki in seinen Arbeiten ausführlich entwickelt und dargestellt worden sind.

Die empfangenen fremdsprachlichen Informationen bestehen einerseits aus den durch die Lernenden im Unterricht erlernten und andererseits aus den ganz neuen/ unbekannten (lexikalischen oder grammatischen) Strukturen, die in den Satzkonstruktionen dargestellt und vermittelt werden. Von jedem Lernenden werden diese Satzkonstruktionen erstens mit Hilfe des analytischen Kodes in kleinere bedeutungstragende Einheiten (Syntagmen und Wörter) segmentiert. Zweitens werden die schon bekannten Strukturen den im fremdsprachlichen Zentrum gespeicherten Matrizen zugeordnet und die neuen Strukturen als Matrizen aufgebaut (Näheres hierzu Zabrocki 1966: 19 ff. u. 1975: 42-52). Um den Inhalt des Satzes zu verstehen, müssen all seine Einheiten zu einem Ganzen zusammengefügt werden. In diesem Falle bedienen sich die Lernenden des synthetischen Kodes. Zusammenfassend bedeutet dies, dass sich die Lernenden während der ganzheitlichen Dekodierung des Satzinhalts zweier Kodes bedienen, des analytischen als des primären Grundkodes und des synthetischen als des sekundären Grundkodes, wobei der letztere auch als Kontrollkode bezeichnet wird. Bei der Erlernung der Fremdsprache eignet man sich zuerst deren phonetisch-phonologischen analytischen Grundkode (den Hörkode) an, dann den phonetisch-phonologischen synthetischen Grundkode (den Sprechkode). So werden auch der graphisch-graphematische analytische Grundkode (der Lesekode) und der graphisch-graphematische synthetische Grundkode (der Schreibkode) beherrscht (Zabrocki 1966: 17, 27 u. 35).

An dieser Stelle dürfte der Hinweis nützlich sein, dass sich sowohl der Hörkode als auch der Lesekode, die auf der ersten Stufe des Dekodierungsprozesses funktionieren, folgendermaßen spezifizieren lassen: 

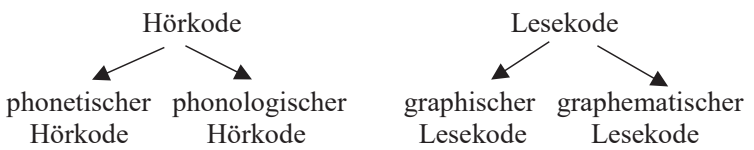

Abbildung 2. Die Aufgliederung des phonetisch-phonologischen analytischen Hörkodes und des graphisch-graphematischen Lesekodes in ihre einzelnen Subkodes

Aus der Spezifizierung beider Kodes ergibt sich, dass für die korrekte Aneignung der fremdsprachlichen bezeichnenden Substanz folgende Bausteine wesentlich sind: zum einen die akustisch-auditiven Eigenschaften der Laute (der Phone) oder die optisch-visuellen Eigenschaften der Schriftzeichen (der Graphe), zum anderen die kleinsten bedeutungsdifferenzierenden Spracheinheiten (die Phoneme oder die Grapheme).

In der Konklusion der Betrachtungen über den Sprachkode-Begriff und die ihm zugrundeliegenden Gesetze weist Zabrocki auf das Wesen des Erlernens einer Fremdsprache hin. Um die Fremdsprache zu beherrschen, um sich deren in den zwischenmenschlichen Kommunikationsakten bedienen zu können, muss man sich zunächst die Kodegesetze der Transformation aneignen. Diese Transformationsgesetze sind Bausteine für die kodematischen und kybernetischen Grundlagen der Theorie des Fremdsprachenunterrichts.

Die Problematik des Fremdsprachenunterrichts ist von vielen Linguisten und Didaktikern erneut aufgenommen worden. Da die Fragen komplex sind und sowohl multidisziplinäre als auch interdisziplinäre Zusammenarbeit betreffen, bedürfen sie selbstverständlich breiterer und gründlicher Überlegungen, wissenschaftlicher Betrachtungen und eingehender Diskussionen über die Erlernungsund Aneignungsprozesse der Fremdsprache(n) (vgl. Hennig \& Huth 1975: 79 ff.).

Moulton und Freudenstein (1972: 39) haben beispielsweise den fremdsprachlichen Gesamtprozess vom kodematischen Standpunkt aus in folgende elf Stufen unterteilt:

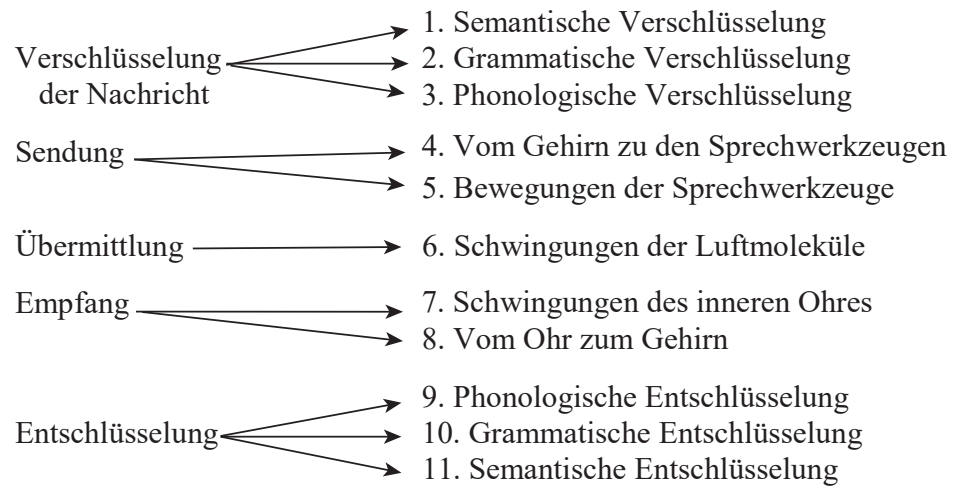


Diese bereits aufgezählten Stufen der Kode-Prozesse verdeutlichen - so Moulton und Freudenstein (1972: 40 ff.) - den ganzen Kommunikationsverlauf, d. h. die inneren und nicht direkt beobachteten Prozesse des Senders und des Empfängers und den äußeren Übermittlungsprozess, der die Kommunikationspartner verbindet. In die semantische Verschlüsselung wird der "Gedanke“, der "Inhalt" einbezogen und als Ausgangspunkt des Prozesses angenommen. Die grammatische Verschlüsselung regelt die entsprechende Anordnung der für die Übertragung von Informationen ausgewählten semantischen Einheiten (Wörter) und deren Kombinationsmöglichkeiten in den Syntagmen und Sätzen. Als letzte findet die phonologische Verschlüsselung statt, in der die syntagmatisch-syntaktisch angeordneten Strukturen phonetisch-phonologisch produziert werden.

In Bühlers (1972: 25) kodematischer Konzeption werden fünf Grundprozesse mit ihren einzelnen Subprozessen folgendermaßen dargestellt:
1) Kodierung der Mitteilung $\longrightarrow$
a) semantische Kodierung
b) grammatische Kodierung
c) phonologische Kodierung
2) Sendevorgang
a) Vom Gehirn zu den Sprechorganen
b) Bewegungen der Sprechorgane
3) Übertragung
Schwingungen der Luftmoleküle
4) Empfangsvorgang
a) Schwingungen des Trommelfells
b) Vom Ohr zum Gehirn
5) Dekodierung
a) phonologische Dekodierung
b) grammatische Dekodierung
c) semantische Dekodierung

Die sprachliche Kodierung versteht Bühler $(27 \mathrm{u}$. 132) als Verwendung der in einer Sprachgemeinschaft festgelegten Zeichen, deren sich die Kommunikationspartner (Sprecher und Hörer) in bestimmten Situationen bedienen. Ein besonderer Wert wird der grammatischen Kodierung beigemessen, weil sie für den Sprechvorgang als strukturierende Komponente anzusehen ist. Um die Eigenart der sprachlichen Kodierung zu beschreiben, sollten vor allem die semantische und die syntaktische Kodierung sowie die Gesprächssituationen berücksichtigt werden.

\section{KODIERUNGS- UND DEKODIERUNGSPROZESSE DER FREMDSPRACHLICHEN INFORMATIONEN}

Den Fremdsprachenunterrichtsprozess beginnt der Lehrende mit der Kodierung der schon erlernten sowie auch der zu lernenden Strukturen, die mit den 
sich schon angeeigneten Strukturen in bestimmte syntagmatisch-syntaktische Konstruktionen eingebaut und gleichfalls dargestellt werden (vgl. auch Grucza 1967: 14f.). Diese Lehrhandlung sollte sich auf das Aneignungsniveau der fremdsprachlichen lexikalisch-grammatischen Strukturen der Lernenden beziehen, über die sie vor allem rezeptiv - jedoch nicht ausschließlich - verfügen, um so alle neuen und bekannten Informationsstrukturen richtig zu dekodieren und die zum ersten Mal vermittelten kennenlernen und als Sprach-Strukturmatrizen im Speicher-Mechanismus aufbauen und behalten zu können. Obwohl das für den Fremdsprachenunterricht aufbereitete Material in den Lehr-Lern-Büchern präsentiert wird und den Unterrichtsteilnehmern zur Verfügung steht, müssen bestimmte Strukturen in den Unterrichtsstunden sowohl (vor allem) mündlich als auch schriftlich vermittelt werden. Dies verlangt auch, dass die Lehrenden feststellen und entscheiden müssen, welche Strukturen des fremdsprachlichen Materials (und warum) lautlich oder graphisch dargeboten werden sollten. An dieser Stelle muss betont werden, dass man im Fremdsprachenunterricht das Primat der Lautsprache vor der Schriftsprache berücksichtigen sollte, was nicht bedeutet, dass das graphische Material im Unterricht vernachlässigt wird. Ganz im Gegenteil, es wird sowohl im Klassenzimmer, aber vor allem im Selbstunterricht als Ausgangspunkt betrachtet und benutzt (Schiffer 1976: 75). Für die fremdsprachenunterrichtlichen Kodierungs- und Dekodierungsprozesse sind also zwei sprachliche Substanzen wichtig, nämlich die lautliche und die graphische, und deshalb werden sie in den weiteren Erörterungen berücksichtigt.

Die glottodidaktischen Kodierungs- und Dekodierungsprozesse werden in das kybernetische Gefüge eingebaut, um zu zeigen, nach welchen Gesetzmäßigkeiten sie ablaufen. Das dargestellte Modell (Abb. 3) umfasst im Allgemeinen die grundlegenden kodematisch-kybernetischen Prozesse, wobei ihre wesentlichen Bestandteile im Einzelnen dargeboten und erklärt werden.

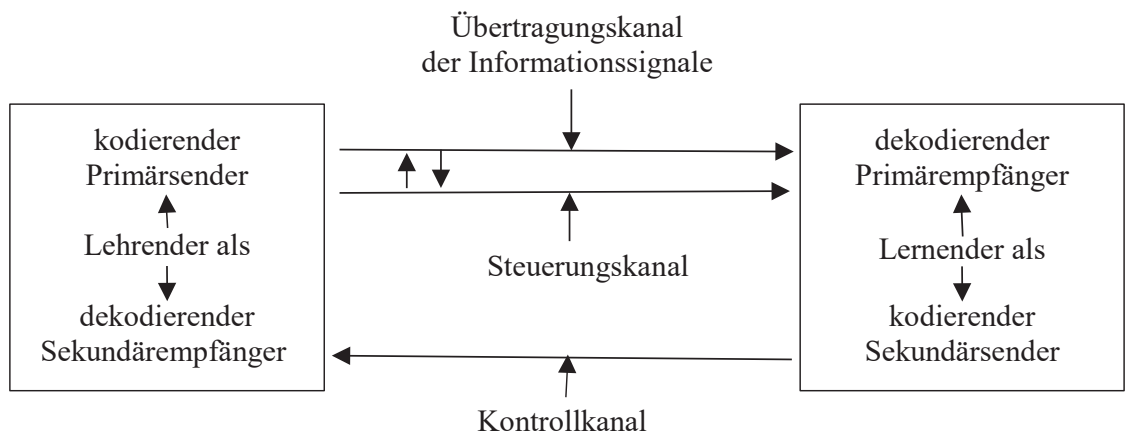

Abbildung 3. Fremdsprachenunterrichtliche Kodierungs- und Dekodierungsprozesse des Lehrenden und des Lernenden mit den interindividuellen Verbindungen 
Wie im Modell angedeutet wird, sind die kodierenden und die dekodierenden Primär- und Sekundärsender im Rückkoppelungsgefüge verbunden. In solch einem Gefüge unterscheidet man drei Schaltungskreise: 1. den Trägerkreis der nuklearen Informationen (Grundinformationskreis), 2. den Steuerungskreis und 3. den Kontrollkreis (Zabrocki 1966: 8 u. 1975: 26). Der Trägerkreis dient der Übertragung der Grundinformationen an die Empfänger/Lernenden. Mit dem Trägerkreis ist direkt und eng der Steuerungskreis verknüpft, der - so wird angenommen - eine inhärente Einheit mit dem Trägerkreis bildet. Seine wesentliche Aufgabe beruht darauf, dass die durch den Sender kodierten Informationssignale von den Empfängern optimal aufgenommen und erfolgreich dekodiert werden. Deshalb benutzt man für die Steuerung der Grundinformationen sowohl die suprasegmentalen Eigenschaften der sprachlichen Strukturen (vgl. die Hervorhebung des Akzents, der Rhythmik oder der Intonation bestimmter Worteinheiten) als auch die parasprachlichen Eigenschaften (Gestik, Mimik) sowie die visuellen Mittel (Bilder, Situationen), welche die entsprechenden Elemente der außersprachlichen Wirklichkeit, die versprachlicht werden sollten, durch Veranschaulichung deutlicher machen. Auf Grund der vom Lernenden kodierten und durch den Kontrollkanal an den Lehrenden überlieferten Informationsstrukturen wird ihre Korrektheit geprüft. Falls einige Strukturen fehlerhaft und/oder mangelhaft kodiert worden sind, bedürfen sie selbstverständlich einer Berichtigung. Welche Sprachstrukturen der Primärsender auswählt und den Lernenden vermittelt, haben wir schon oben angedeutet. Seine intraindividuellen Kodierungsprozesse vollziehen sich im fremdsprachlichen Speicher-Mechanismus, wo die lexikalisch-grammatisch bekannten Strukturen und eine neu zu lernende lexikalische oder grammatische Struktur generiert und syntagmatisch-syntaktisch angeordnet werden. Fungiert der Primärsender als Sprecher, so werden die syntagmatisch-syntaktisch generierten Strukturen in neuronale Substanz umgewandelt und in Artikulationseffektoren transportiert, wo deren artikulatorische Produktion stattfindet. Auf diese Weise vollzieht sich der intraindividuelle Kodierungsprozess, der aus zwei Subprozessen (der Generierung und der Produktion) besteht. Demnach wird die artikulatorische Substanz der kodierten Strukturen im interindividuellen Übertragungskanal in akustische Signale transponiert und den Rezeptoren der Empfänger/Lernenden übermittelt, wo sie in auditive Signale der phonetisch-phonologischen Strukturen umgewandelt werden.

Agiert der Primärsender als Scheiber, werden die im fremdsprachlichen Speicher-Mechanismus generierten Strukturen in neuronale Substanz umgewandelt und zu motorischen Effektoren transportiert, wo deren motorisch-graphische Produktion stattfindet. Im Übertragungskanal wird die motorisch-graphische Substanz in optische Signale transponiert und den Rezeptoren der Empfänger/ 
Lernenden übermittelt, wo sie in visuelle Signale umgewandelt werden. In den Rezeptoren beginnt die erste Stufe des Dekodierungsprozesses, während dessen die phonetisch-phonologischen Strukturen differenziert, in neuronale Substanz transponiert und zum Speicher-Mechanismus transportiert werden. Dort findet die zweite Stufe des Dekodierungsprozesses statt, nämlich der ganzheitliche, d. h. der lexikalisch-grammatische Dekodierungsprozess. Da die durch den Primärsender/Lehrenden kodierten und vermittelten Informationsstrukturen aus bekannten und neu dargebotenen fremdsprachlichen Struktureinheiten bestehen, werden die bekannten den schon im Speicher-Mechanismus des Lernenden existierenden Sprachmatrizen zugeordnet und so verstanden, die neuen dagegen werden dekodiert und kennengelernt sowie als Sprachmatrizen konstruiert und dort aufbewahrt. Hierzu sei noch darauf verwiesen, dass die Dekodierung als zweistufiger Prozess betrachtet wird, in dem die neuen fremdsprachlichen Struktureinheiten als Matrizen konstruiert und im Gedächtnis aufbewahrt oder gespeichert werden, die bekannten Struktureinheiten hingegen durch ihre Dynamisierung eine festere Einlagerung gewinnen, die sicherlich das Funktionieren des Speicher-Mechanismus ermöglicht. Unter dem Begriff der fremdsprachlichen Struktureinheiten versteht man im glottodidaktischen Sinne die grundlegenden Spracheinheiten, nämlich die lexikalischen Strukturen und die grammatischen Strukturen, die des Weiteren auf lautliche oder schriftliche Art und Weise im Fremdsprachenunterricht dargeboten und erlernt werden.

Die durch die Lernenden neu empfangenen und dekodierten fremdsprachlichen Einheiten hinterlassen im Gedächtnis Spuren, auch Engramme genannt (vgl. u. a. Parreren 1972: 297ff.; Gagne 1973: 67; Schnabl 1972: 131; Hoffmann \& Klix 1980: 192; Wettler 1980: 12), die unter kodematischem Aspekt als SprachStrukturmatrizen bezeichnet werden. Im Falle der lautlichen Vermittlung der neuen lexikalischen oder grammatischen Struktur und deren phonetisch-phonologischer Dekodierung wird im Speicher-Mechanismus jedes Lernenden die Sprech-Sprach-Strukturmatrize konstruiert, während der schriftlichen Vermittlung und deren graphisch-graphematischer Dekodierung wird dagegen die Schreib-Sprach-Strukturmatrize aufgebaut. Wenn die lexikalische SprechSprach-Strukturmatrize im Speicher-Mechanismus des Lernenden vorhanden ist und im Lernprozess deren Schreib-Sprach-Strukturmatrize konstruiert wird, dann kommt es zur völligen Verkoppelung beider Sprach-Strukturmatrizen in eine in sich geschlossene und übergeordnete bezeichnete Ganzheit, die begrifflich als Sprech-Schreib-Sprach-Strukturmatrize erfasst werden kann. Daraus ist der glottodidaktische Schluss zu ziehen, dass die fremdsprachlichen Strukturmatrizen durch den Lernenden primo als Sprech-Sprach-Strukturmatrizen, secundo als Schreib-Sprach-Strukturmatrizen und tertio - wenn die Sprachstrukturen lautlich und schriftlich parallel dargeboten und dekodiert werden - als Sprech-Schreib- 
Sprach-Strukturmatrizen aufgebaut werden und so funktionieren können. Die bisherigen Erörterungen bezüglich der Sprach-Strukturmatrizen lassen sich modellhaft wie folgt konzipieren:

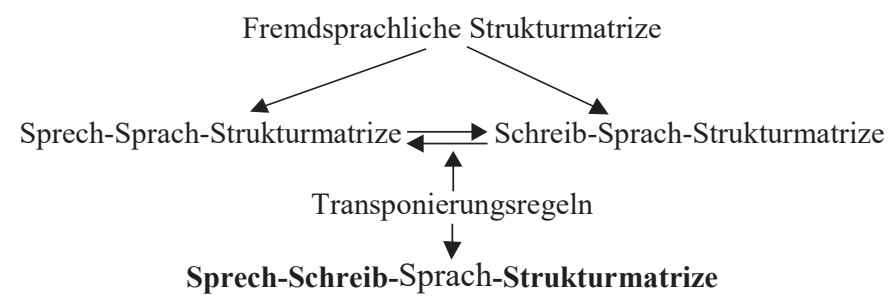

Abbildung 4. Die Verkoppelung der Sprech-Sprach-Strukturmatrize und der Schreib-SprachStrukturmatrize in die Sprech-Schreib-Sprach-Strukturmatrize

Die neu lexikalisch oder grammatisch konstruierten Sprech-Sprach- und Schreib-Sprach-Strukturmatrizen bedürfen sicherlich vielfältiger Präzisierung, weil deren Aufbau erstens bei jedem Lernenden durch seine individuellen Lernfähigkeiten bedingt ist, was einen direkten Einfluss auf deren Aufbewahrungszeit im Speicher-Mechanismus hat, und zweitens sich die Sprach-Strukturmatrizen hinsichtlich der rezeptiven, reproduktiven und produktiven Leistungsfähigkeiten (ganz) gewiss bei jedem Lernenden unterscheiden. Drittens müssen auch die Verbindungsmöglichkeiten der neu erlernten mit den bekannten Strukturen in syntagmatischen und syntaktischen Konstruktionen berücksichtigt werden.

Mit den bereits aufgeworfenen Fragen ist der Kodierungsprozess des Fremdsprachenlernenden verbunden, in dem er als selbständiger Empfänger, aber insbesondere als Sender in den Kommunikationsakten fungiert. Seine fremdsprachlichen Handlungen basieren auf den im Speicher-Mechanismus vorhandenen lexikalischen und grammatischen Strukturen, die als Sprecheinheiten oder Schreibeinheiten benutzt werden. Diese Einheiten bilden das fremdsprachliche Inventar, auf Grund dessen die benötigten Informationen kodiert werden. Um die Informationen zu kodieren, finden im Speicher-Mechanismus des Lernenden folgende Subprozesse statt: 1. Der Suchprozess der für die Kommunikationsintention entsprechenden Wortstrukturen, 2. Der Auffindungsprozess der für die Kodierung benötigten Wortstrukturen, 3. Der Transformationsprozess der ausgewählten Wortstrukturen und deren Anordnung in die syntagmatischen und syntaktischen Konstruktionen und 4. Der Abrufprozess oder der Generierungsprozess der angeordneten Wortstrukturen, die des Weiteren zu den artikulatorischen oder den motorischen Effektoren transportiert werden, wo sich deren Produktionsprozess vollzieht. Der Generierungs- und der Produktionsprozess sind Bestandteile des Kodierungsprozesses der Informationsstrukturen (vgl. Szczodrowski 2001:145-165). 


\section{EINKODIERUNGSPROZESSE DER FREMDSPRACHLICHEN STRUKTURMATRIZEN}

Dass die fremdsprachlichen Strukturen durch den Lernenden dekodiert und im Speicher-Mechanismus als konstruierte Sprech-Sprach-Schreibmatrizen aufbewahrt werden, bedeutet bei weitem noch nicht, dass sie dem Lernenden/ Fremdsprachenbenutzer auf unbegrenzte Zeit zur Verfügung stehen werden. Sie bedürfen ständiger rezeptiver, reproduktiver und vor allem produktiver Dynamisierungen, die dazu beitragen, dass sie ins Langzeit-Gedächtnis übertragen, dort tiefer und fester verankert werden und dadurch ihre operations- und leistungsfähige rezeptiv-produktive Potenz erreichen. Solch einen Übertragungsprozess der konstruierten Sprach-Strukturmatrizen ins Langzeit-Gedächtnis bezeichnen wir als Einkodierungsprozess (= Einprägungsprozess, vgl. dazu Szczodrowski 2016), wo sein Wesen und seine Eigenschaften im Bereich der kodematischen Theorie reflektiert und präzisiert worden sind.

Auf Grund der Dekodierung der neu empfangenen fremdsprachlichen lexikalischen oder grammatischen Strukturen vollzieht sich der Aufbau deren Matrizen im Gedächtnis des Lernenden, die dort für eine gewisse Zeit verfügbar sind. An dieser Stelle erhebt sich einerseits die Frage, die in einem direkten Zusammenhang mit dem spatio-temporalen Charakter der fremdsprachlichen Matrizenkonstruktion im Gedächtnis steht. Das Gedächtnis ist bekanntlich die Grundlage der Lernprozesse und diesbezüglich umfasst es die Aufnahme, das Behalten und die Wiedergewinnung der (fremd-)sprachlichen Informationen. Da sich im Gedächtnis des Menschen sowohl das Zentrum der Muttersprache als auch der Raum der neu konstruierten fremdsprachlichen Matrizen befindet, bezeichnen wir ihn als Speicher-Mechanismus. Dort werden die bereits erwähnten Matrizen für eine bestimmte Zeit aufbewahrt (im Speicher) und sind dementsprechend mit der Vorrichtung der Rezeptions-, Reproduktions- und Produktionspotenz (des Mechanismus) ausgestattet. Andererseits besteht noch eine offene Frage, und zwar wie lange die fremdsprachlichen Sprachstrukturmatrizen im Speicher behalten werden. Die Aufbewahrungsdauer dieser Matrizen ist nicht unbegrenzt und deshalb berücksichtigen wir das möglichst dreistufige Behalten der konstruierten fremdsprachlichen Matrizen im Gedächtnis des Lernenden (vgl. dazu Vester 1975: 58 u. passim), nämlich deren

- Aufbewahrung im Ultrakurzzeit-Gedächtnis (UKZG)

- Speicherung im Kurzzeit-Gedächtnis (KZG) und

- Einkodierung im Langzeit-Gedächtnis (LZG)

Wesentlich für jeden Lernprozess sind insbesondere zwei Probleme: das Behalten des Erlernten und dessen Verlust. Die Lernprobleme sind nicht identisch für jeden Lernenden, weil die rezeptive und die produktive Aneignung des 
fremdsprachlichen Systems - sowohl des phonetisch-phonologischen als auch des lexikalisch-grammatischen - durch die Sprachbegabung jedes Individuums in irgendwelchem Maße bedingt ist. Um die im UKZG aufbewahrten fremdsprachlichen Sprachstrukturmatrizen länger zu behalten sowie die im KZG gespeicherten ins LZG zu übertragen, verlangen die Sprachstrukturmatrizen systematische rezeptive und (re-)produktive Dynamisierungen. Verzichtet man auf die bereits genannten Übertragungen der Sprachstrukturmatrizen, so zerfallen sie wahrscheinlich ganz und gar. Mit den rezeptiv-produktiven fremdsprachlichen Handlungen ist auch die Verarbeitung des gelernten Materials verbunden, die beispielsweise durch die Verwendung der Wortstrukturen in den syntagmatisch-syntaktischen Konstruktionen zustande kommt, und zwar in den Äußerungen der Lernenden während des Unterrichts, in den schriftlichen Übungen im Selbstunterricht und auch in den möglicherweise vorkommenden selbstständigen Kontakten mit den fremdsprachlichen Kommunikationspartnern.

Das LZG scheint der dauerhafteste Speicher zu sein und deshalb haben wir auf die Eigenschaften des Einkodierungsprozesses hingewiesen, auf Grund dessen die fremdsprachlichen Sprachstrukturmatrizen tiefer ins Gedächtnis eingeprägt werden und dort eine sichere Existenzgrundlage erhalten sowie operationsfähiger funktionieren können. Der Einkodierungsprozess basiert auf den bereits erwähnten Dynamisierungen der im UKZG aufbewahrten und der im KZG gespeicherten fremdsprachlichen Strukturmatrizen und deren Übertragung ins Langzeit-Gedächtnis, wo ihre Existenz zwar nicht unendlich ist, aber wo sie ganz gewiss einen längeren Zeitraum überdauern. Alle Dynamisierungen der im LZG einkodierten Strukturmatrizen festigen in verschiedenartigen Kommunikationsakten ihre bezeichnenden und bezeichneten Substanzen und deren Leistungsfähigkeiten, die auch davon abhängig sind, in welchen Schichten des Langzeit-Gedächtnisses sie verankert worden sind. Hierzu wird ein Modell konzipiert (Abb. 5, vgl. auch Szczodrowski 2018: 350), welches die mögliche Verteilung der fremdsprachlichen Sprech-Schreib-Strukturmatrizen in diesem Gedächtnis darstellt:

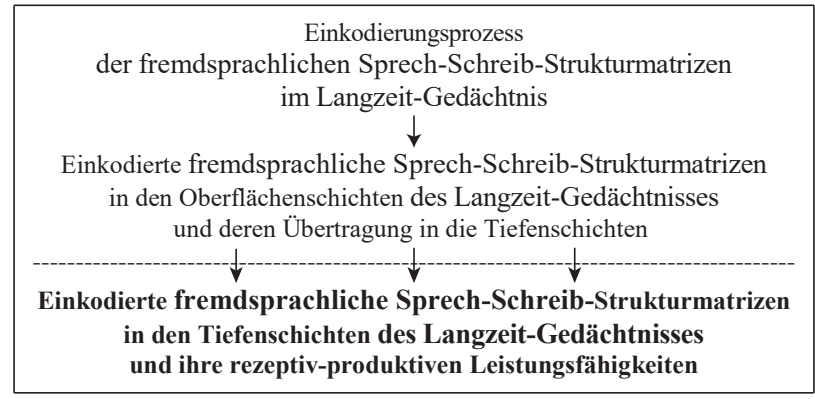

Abbildung 5. Übertragung der einkodierten fremdsprachlichen Sprech-Schreib-Strukturmatrizen von den Oberflächenschichten in die Tiefenschichten des Langzeit-Gedächtnisses 
Da die einkodierten fremdsprachlichen Sprech-Schreib-Strukturmatrizen im Langzeit-Gedächtnis jedes Lernenden/Benutzers hinsichtlich der räumlich-zeitlichen und der operationsfähigen Eigenschaften nicht gleich sind, haben wir im Speicher-Mechanismus deren zwei Schichten differenziert, in denen sie als interiorisierte Strukturmatrizen ihre Dauerhaftigkeit und ihr kommunikatives Leistungsvermögen erzielen. Betrachtet man den Fremdsprachenlernenden hinsichtlich des effektiven Einkodierens der einzelnen fremdsprachlichen Struktureinheiten, dann sollten ebenso folgende Probleme berücksichtigt werden: Die Spezifik der lexikalisch-grammatischen Strukturen und deren lautliche oder schriftliche Realisierung, die nötige Einprägungszeit und die erreichten Leistungen. Dass manche im Langzeit-Gedächtnis einkodierten fremdsprachlichen Strukturmatrizen irgendwann auch in (völlige) Vergessenheit geraten (können), kommt aus verschiedenen Gründen nicht selten vor.

\section{ZUSAMMENFASSUNG}

Abschließend taucht das linguistische und das glottodidaktische Problem auf, wie die kodematische Theorie bezüglich der sich im Fremdsprachenunterricht vollziehenden Prozesse einerseits durch die Lehrenden und andererseits durch die Lernenden in die Praxis umgesetzt werden kann. Betrachtet man unter dem glottodidaktischen Aspekt die vorgenommene Einteilung der im Unterricht stattfindenden Kode-Prozesse noch einmal, so fällt auf, dass sowohl der Lehrende wie auch der Lernende als Primärsender und Primärempfänger sowie als Sekundärsender und Sekundärempfänger bestimmte fremdsprachliche Aufgaben zu erfüllen haben, um die gesetzten Ziele zu verwirklichen. Wenn die Lernenden an den bereits erwähnten Kommunikationshandlungen teilnehmen (wollen), müssen sie sich die Strukturen sowie deren richtige Anordnung in den syntagmatisch-syntaktischen Konstruktionen angeeignet haben.

Insbesondere wurden auch das Wesen des Einkodierungsprozesses der fremdsprachlichen Sprech-Schreib-Strukturmatrizen im Langzeit-Gedächtnis des Lernenden und dessen Ergebnisse betont. Jede Dynamisierung der Strukturmatrizen versteht sich als deren Aktualisierung in einer bestimmten Kommunikationshandlung, was naturgemäß dazu beiträgt, dass die Strukturmatrizen tiefer im Langzeit-Gedächtnis des Lernenden verankert sind, dass sie sich durch eine längere räumlich-zeitliche Stabilität auszeichnen, was eine direkte Wirkung auf die rezeptiv-produktiven Leistungsfähigkeiten und -fertigkeiten des Lernenden ausübt. 


\section{LITERATURVERZEICHNIS}

Baacke, D. (1973). Kommunikation und Kompetenz. Grundlegung einer Didaktik der Kommunikation und ihrer Medien. München: Juventa Verlag.

Bühler, H. (1972). Sprachbarrieren und Schulanfang. Weinheim et al.: Beltz Verlag.

Bünting, K.-D. / Kochan, D.-C. (1973). Linguistik und Deutschunterricht. Kronberg: Scriptor Verlag $\mathrm{GmbH} \&$ Co KG Wissenschaftliche Veröffentlichungen.

Gagne, R.M. (1973). Die Bedingungen des menschlichen Lernens (aus dem Amerikanischen übersetzt von Helmut Skowronek). Hannover et al.: Hermann Schroedel Verlag KG.

Gipper, H. (1978). Sprachwissenschaftliche Grundbegriffe und Forschungsrichtungen. München: Max Hueber Verlag.

Grucza, F. (1967). Metasprache, Kodematik, Fremdsprachenunterricht. Glottodidactica. An International Journal of Applied Linguistics, 2, 11-20.

Helbig, G. (1970). Gesichte der neueren Sprachwissenschaft. Leipzig: VEB Bibliographisches Institut.

Hennig, J. / Huth, L. (1975). Kommunikation als Problem der Linguistik. Göttingen: Vandenhoeck \& Ruprecht.

Hoffmann J. / Klix, F. (1980). Zur Prozeßcharakteristik der Bedeutungserkennung über sprachlichen Reizen. In: M. Bierwisch (Hrsg.), Psychologische Effekte sprachlicher Strukturkomponenten (S. 315-374). München: Wilhelm Fink Verlag.

Moulton, W.G. / Freudenstein, R. (1972). Wie lernt man fremde Sprachen? Dortmund: Verlag Lambert Lensing $\mathrm{GmbH}$.

Parreren, C.F. van (1972). Lernprozeß und Lernerfolg. Braunschweig: Georg Westermann Verlag.

Schiffer, L. (1976). Einführung in den audio-visuellen Fremdsprachenunterricht. Heidelberg: Quelle \& Meyer.

Schnabl, H. (1972). Sprache und Gehirn - Elemente der Kommunikation. Zu einem kybernetischen Modell der menschlichen Nachrichtenverarbeitung. München: Wilhelm Goldmann Verlag.

Szczodrowski, M. (2001). Steuerung fremdsprachlicher Kommunikation. Gdańsk: Wydawnictwo Uniwersytetu Gdańskiego.

Szczodrowski, M. (2016). Zum Wesen der fremdsprachlichen Einkodierungsprozesse. Glottodidactica. An International Journal of Applied Linguistics, 43 (1), 69-80.

Szczodrowski, M. (2018). Zum Begriff der Einkodierung fremdsprachlicher Strukturen. Studia Niemcoznawcze, 62, 339-356.

Vester, F. (1975). Denken, Lernen, Vergessen. Stuttgart: Deutsche Verlags-Anstalt.

Wettler, M. (1980). Sprache, Gedächtnis, Verstehen. Berlin et al.: Walter de Gruyter.

Zabrocki, L. (1961). Sprachkode. Zeitschrift für Phonetik, allgemeine Sprachwissenschaft und Kommunikationsforschung, 14 (1), 64-73.

Zabrocki, L. (1966). Kodematische Grundlagen der Theorie des Fremdsprachenunterrichts. Glottodidactica. An International Journal of Applied Linguistics, 1, 3-42.

Zabrocki, L. (1975). Kybernetische Modelle der sprachlichen Kommunikation. Wrocław et al.: Zakład Narodowy im. Ossolińskich, Wydawnictwo Polskiej Akademii Nauk, Komitet Neofilologiczny.

Received: 14.04.2020; revised: 10.10 .2021

MARIAN SZCZODROWSKI

Ateneum Szkoła Wyższa w Gdańsku

m.szczodrowski6@upcpoczta.pl

ORCID: 0000-0003-4881-8226 
